\title{
Security Policy Reconciliation in Distributed Computing Environments
}

\author{
Hao Wang, ${ }^{*}$ Somesh Jha ${ }^{\dagger}$, Miron Livny* \\ Computer Sciences Department \\ University of Wisconsin \\ Madison, WI 53706 \\ \{hbwang, jha, miron\}@cs.wisc.edu
}

\author{
Patrick D. McDaniel \\ AT\&T Labs-Research \\ Shannon Laboratory \\ 180 Park Ave., Rm. A203 \\ Florham Park, NJ 07932 \\ pdmcdan@research.att.com
}

\begin{abstract}
A major hurdle in sharing resources between organizations is heterogeneity. Therefore, in order for two organizations to collaborate their policies have to be resolved. The process of resolving different policies is known as policy reconciliation, which in general is an intractable problem. This paper addresses policy reconciliation in the context of security. We present a formal framework and hierarchical representation for security policies. Our hierarchical representation exposes the structure of the policies and leads to an efficient reconciliation algorithm. We also demonstrate that agent preferences for security mechanisms can be readily incorporated into our framework. We have implemented our reconciliation algorithm in a library called the Policy Reconciliation Engine or PRE. In order to test the implementation and measure the overhead of our reconciliation algorithm, we have integrated PRE into a distributed highthroughput system called Condor.
\end{abstract}

\section{Introduction}

Security policy bridges the gap between static implementations and the broad and diverse security requirements of user communities. Security policy becomes more complicated in heterogeneous environments. When two or more

\footnotetext{
*Hao Wang and Miron Livny were supported by DOE under contract DE-FC02-01ER25464 and NSF/NASA under contract 795NAS1115A.

$\dagger$ Somesh Jha was supported by Office of Naval Research under contracts N00014-01-1-0796 and N00014-01-1-0708.

The U.S. Government is authorized to reproduce and distribute reprints for Governmental purposes, notwithstanding any copyright notices affixed thereon. The views and conclusions contained herein are those of the authors, and should not be interpreted as necessarily representing the official policies or endorsements, either expressed or implied, of the above government agencies or the U.S. Government.
}

entities share a security association, they must reach agreement on a governing policy (e.g., two end-points in an IPsec session). These entities express their requirements for the association through a security policy (called a domain policy). A reconciliation algorithm finds a policy that is consistent with all domain policies. Where a consistent policy can be found, the association is free to proceed. Where one cannot be found, the participants must alter their requirements or abstain from participating.

In the general case, policy reconciliation is intractable [15, 23]. As a result, past investigations have largely achieved tractability by limiting the policy representation or by using heuristic algorithms [11, 24, 26, 33]. Such approaches achieve the stated goals, but fail to efficiently capture dependencies between different aspects of a policy. Moreover, these systems do not consider preferential policy i.e., it is advantageous (and often necessary) for policy not only to specify what is legal and illegal, but to state what is desirable.

This work addresses the limitations of past work by developing a policy framework based on graphical policy representations. We exploit the graph representation to efficiently encode the complex dependencies inherent to contemporary policy. We formally define the representation and specify an efficient preference and dependencyrespecting reconciliation algorithm. Before introducing our formalism, we present an overview of security provisioning policy and the intuition behind our framework in the following section.

\subsection{Security Policy}

The term security policy has come to mean different things to different communities. For example, access control policy defines who has access to what and under what circumstances [4, 29, 30]. Other forms of security policy specify under what conditions credentials are accepted [6], 


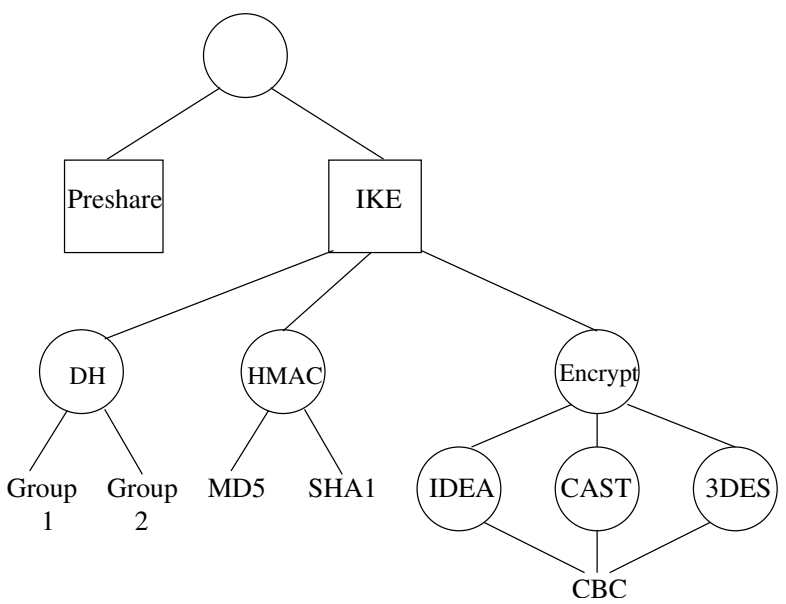

Figure 1. A graphical IPsec key management policy

or how a firewall is configured [3]. In its broadest definition, security policy is the specification of security relevant system behavior. This paper addresses session-specific configuration of security services. More commonly known as security provisioning policy, these configurations define the guarantees afforded the governed environment by explicitly identifying the algorithms, parameters, and protocols used to implement security.

To illustrate the importance and ubiquity of security provisioning policy, consider an email client (e.g., Netscape Communicator, MS Outlook). A user specifies a provisioning policy every time she adds an account. For example, the connection method (e.g., IMAP over SSL) dictates exactly the set of guarantees you will receive in obtaining and viewing your mail. Note that the decision to not use any security service is still a specification of policy. The policies defined for the applications and services used in an environment prescribe the security afforded its users.

In practice, provisioning policy is more complicated than our email example would suggest. It is often important that particular organization-wide goals are realized in the many policies implemented by the environment. Lower level policies must be constructed such that they are compliant with organizational goals [23]. Moreover, where an operation spans organizations, the policies of each organization must be reconciled to form a coherent and reasonable policy.

We now introduce our graphical provisioning policy representation. A graphical policy is a series of policy operations represented by cascading circular or square nodes in a singularly rooted directed acyclic graph (DAG) (formally this structure is an and-or graph). Policy is read from the root node. Each node may be a decision (circle) or a collection (square). A decision node requires that exactly one of the sub-graphs emanating from the node be resolved, and a collection node requires all sub-graphs be resolved. All leaf nodes are added to the policy. Any configuration derived from a policy respects these two simple rules.

Figure 1 shows a graphical provisioning policy for key management used in an IPsec VPN. This policy would be specified by a user or network administrator as part of, for example, VPN setup. One reads the example policy's root (decision node) as:

(configure) Preshare (preshareed keys) or IKE

The right-hand side of the graph (IKE, from the root) depicts a complex series of configurations used to specify the behavior of the Internet Key Exchange (IKE) protocol [16]. The IKE sub-policy consists of three independent configurations. We read the top IKE (collection node) as:

\section{(configure) DH group and HMAC and Encryption}

The remainder of the policy is read as a selection of a single DH group, a hashing algorithm, and an encryption algorithm. Independent of the encryption algorithm, a mode (e.g., CBC) must be selected. Moreover, this policy mandates the use of $\mathrm{CBC}$ mode.

The example policy is used at the point at which an endpoint (host) is connected to the VPN. The policy is evaluated by identifying a subset of nodes and leaves in graph as defined by the structure of the collection and decision nodes. The IPsec implementation uses the resulting concrete specification, called an evaluated policy or instance, to implement the IPsec session. For example, one possible evaluated policy contains: IKE, DH-Group 2, HMAC-MD5, IDEA-CBC.

Two important factors are highlighted by this example. First, this is one of many possible policies for IPsec key management. Depending on the goals of the specified policy, the specifier may structure the policy in a number of different ways. For example, inasmuch as it is consistent with the IPsec implementation, the policy can allow other encryption modes (e.g., ECB) by adding an additional decision node.

The second factor of note is that unlike our email policy, this policy specifies a range of behaviors. That is, the policy states that there are a set of configurations that are equally acceptable. The structure of the graph directly mandates which sets of configurations should be considered acceptable. Having non-prescriptive policies allow the environment to make performance and security trade-offs at run time, and is essential to reconciling policies from different domains.

Now consider the case where there is not a single source of policy: for example, where the end-points of the VPN lie in different administrative domains. Each domain wishes to exert control over the session as specified through a domain policy (e.g., similar to Figure 11. Hence, the two parties must find an evaluated policy that is consistent with the do- 
main policies supplied by both. This is performed by reconciling the domain policies. The session can continue only where a single governing policy can be found. If not, the domain policies are incompatible and the end-points must alter their policies or refrain from participating in the session.

The study of provisioning policy is unlike other policy efforts in several ways. First and most obviously, provisioning policy is a planning process. Traditional authorization policy systems determine whether a particular access is legal with respect to some larger governing policy. Conversely, provisioning policy attempts to find some configuration that is consistent with a governing provisioning policy.

Provisioning policy also embodies complex dependencies. That is, decisions about particular aspects of the policy affect subsequent options. Figure 1 illustrates a very simple dependency: the decision to use IKE over pre-shared keys has enormous impact on the further development of policy. The selection of IKE leads to decisions concerning the kinds of Diffie-Hellman groups to use, what encryption algorithms are necessary, etc. However, if pre-shared keys were selected, other configuration values (e.g., DiffieHellman group) should and would not be considered.

Provisioning is also subject to preferential behavior. That is, there is a often a set of configurations that is most desired among several choices. Again consider Figure 1 According to the policy, either group 1 or group 2 is acceptable. In practice, we have found the vast majority of IPsec configurations use group 2. As such, we (rightly or wrongly) may decide that group 2 is best for our environment, and is thus preferred. However, for compatibility reasons, we do not wish to preclude the use of group 1. Note that preferential configurations are more than simple default values, but a partial ordering of the available options. The existence of preferences is largely ignored by previous work in this area.

As we demonstrate in the following sections, reconciliation is made more complex by the introduction/appreciation of these deeper aspects of policy. While this work aspires to provide intuitive policy representations, it must do so within the constraints of these new complex semantics. Hence, our contribution lies not only in the representation or added semantics, but in the successful marriage of the two.

\subsection{Contributions}

This paper addresses the aforementioned deficiencies of existing systems by modeling dependencies and preferences in a graphical policy framework. The main contributions of this paper are:

-Graph-based provisioning policy (exposes dependencies): We present a model that represents policies as directed acyclic graphs (DAG). This model captures dependencies between policy components within a schema.
Hence, because policies adhere to the schema, it is impossible to define a correctly formed policy that is not consistent with the dependencies.

-Efficient reconciliation: In general, policy reconciliation is $N P$-complete [23]. However, a graphical representation of policies expose their structure and present a basis for an efficient reconciliation algorithm. We provide an efficient reconciliation algorithm for our graphical model. Our reconciliation algorithm is linear in the size of the policies.

-Preferential policy: Participant preferences, such as a server's preferences for authentication mechanisms, can be incorporated into our model. An important problem that arises in this context, is that of resolving multiple partial orders on the same set (intuitively, these partial orders represent preferences of different participants). We provide an efficient algorithm to resolve multiple partial orders and extend the reconciliation algorithm to handle preferences.

-Implementation and deployment: Based on our hierarchical framework, we have implemented a reconciliation module called the Policy Reconciliation Engine or PRE, which is available for download. We have integrated PRE with Condor [21], a high-throughput scheduling system used to manage resources in a complex distributed environment. We show experimentally that the cost of reconciliation is negligible.

\section{Related Work}

Other policy systems. Historically, policy systems have not addressed reconciliation. For example, trust management systems, such as KeyNote [5], SPKI/SDSI [12, 13], Binder [10], and SD3 [18] are concerned with compliance checking rather than reconciliation. In trust management systems, policies, called credentials, are simply cryptographic proofs that express authorization delegation. The compliance checker algorithm searches the available credentials for an accepting delegation chain that satisfies a specific request. Credentials can state a set of provisioning requirements. An action is only allowed where the provisioning of the environment matches the credential. Such approaches are useful for managing policy in a widely deployed or loosely organized environments [7]. However, because credentials mandate provisioning, there is no opportunity to perform reconciliation. Other systems simply assume a singular entity manually performs reconciliation when issuing policy for a domain [3].

Hardness of reconciliation. While reconciliation has only recently begun to be explored, the policy community has already developed a broad characterization of the problem. Gong and Qian discovered that reconciliation of authorization policy (in their work, called policy composition) is 
NP-complete [15]. Similarly, the authors of Ismene found that reconciliation of general purpose provisioning policy is also NP-complete [23]. Such results do not mean that progress cannot be made, but suggests a required shift in the goals of investigation. Much of the ongoing work in reconciliation has centered on techniques that alter the environment or restrict policy to obtain efficient reconciliation. However, our paper demonstrates that by using a representation that exposes structure of the policies, the reconciliation problem becomes tractable for a larger class of policies.

Other reconciliation approaches. One way to address the inherent complexity of reconciliation is by essentially "flattening" the policy representation, i.e., explicitly enumerating the various choices. For example, the IPsec Security Policy System (SPS) [33] guarantees efficient two-party reconciliation by intersecting fixed and independent sets of policy values. The DCCM system extends this approach to the multi-party environments by providing a Chinese menu reconciliation algorithm [1,2,11]. Each participant chooses values from a fixed set of policy dimensions (e.g., one from column $A$, two from column, $B$, etc ...). The policy is reconcilable where an intersection of proposals is found for each dimension. Conflicts (where no such intersection is found) are resolved by an unspecified algorithm.

A limitation of both SPS and DCCM is that they assume that there are no dependencies between policy values. For example, in an IPsec policy, an encryption algorithm is needed when the ESP transform is selected. Therefore, to ensure that the resulting policy is enforceable, one must disallow any policy that defines the ESP transform but no encryption algorithm. In practice, these systems define policy as an enumeration of legal policy combinations, such as ESP-3DES-HMAC-SHA. Since only legal enumerations are available, no dependency can be violated. However, the number of enumeration values grows exponentially in the size of the domains, and therefore the "enumeration approach" is inherently not scalable.

Ismene policies are defined as expressions of provisioning variables [23]. The reconciliation algorithm tries to find a satisfying truth assignment for the universe of provisioning variables. Reconciliation is cast as an instance of satisfaction (over the conjunction of policy proposals). Efficiency is guaranteed by using a pair-wise satisfaction algorithm on restricted policy expressions. The iterative Ismene $n$-policy reconciliation algorithm is sound but not complete, i.e., some collections of reconcilable policies may be rejected. Furthermore, like SPS, the Ismene reconciliation algorithm does not consider dependencies. Dependencies are addressed in Ismene by evaluating the reconciliation result with respect to a set of "correctness rules" using an analysis algorithm. This approach is limited in that it occurs after the policy has been identified. Hence, reconciliation must be re-performed after each policy is rejected by analysis.
BANDS [32] addresses multi-domain policy reconciliation in the context of IPsec by describing the security requirements of each domain in a policy language [14]. The provisioning policy between two nodes (source and destination) is proposed by the source node through a gathering phase where security requirements from all domains along the path are gathered. From the gathered requirements, a policy is then proposed and passed along the path to the destination node. Each domain along the path must verify the policy against its own security requirements or return an error to the source node. If the proposed policy reaches the destination node without an error, it becomes the provisioning policy for the session.

A central limitation of the approaches defined above is that they are not sensitive to the structure of policy. Dependencies between different aspects of policy are either inefficiently encoded or externally evaluated. This is a prime motivation of the current work. Dependencies are captured through the graphical structure of the policy schema, and hence any policy resulting from reconciliation is guaranteed to be consistent with these dependencies. Previous reconciliation algorithms also make no distinction between reconciliation results. Since no distinction is made, every possible result is equally desirable. However, environments often desire to specify default behavior and allow others where the defaults are inefficient or infeasible. This work allows such desires to be expressed through preferences.

Other work on representation and analysis of security policies. Cholvy and Cuppens consider the complexities of detecting and managing inconsistencies introduced by access control policy specifications [8]. Our approach differs not only in problem domain (i.e., provisioning), but in that we avoid consistency evaluation by encoding dependencies within the policy structure. Hence, collections of individual policies cannot be inconsistent. Cholvy and Cuppens further considered preference in the context of the ordered application of access control regulations, but focused on access control applications.

While it has not been explored for other forms of policy, graphical representations are well suited to access control policy [20, 25]. For example, the LaSCO language specifies access control policy using graphical idioms [17]. The developers of $\mathrm{LaSCO}$ assert that the representation allows not only specification a more intuitive operation, but permits the use of well known graph algorithms for subsequent enforcement. We embrace a similar approach by using structural representation to enforce dependencies.

\section{A Formalization of Policy Reconciliation}

In this section we provide a precise semantics of policy reconciliation where the policies are represented hierarchically. Moreover, we describe how preferences can be incor- 




none X.509 KerberosPassword none 3DES AES IDEA SAFER

Figure 2. Schemata $S$

porated into our framework. Finally, we present an efficient reconciliation algorithm.

Definition 3.1 A schemata is a directed acyclic graph or DAG $S=(N, E$, root $)$, where $N$ is a set of nodes, $E \subseteq$ $N \times N$ is a set of edges, and root $\in N$ is a distinguished node. We assume that root has no incoming edge. Each node $n$ has the following attributes associated with it:

- Each node is a $\wedge$ or $\vee$ node.

- A tuple of variables (denoted by $\operatorname{Var}_{T}(n)$ ) $\left\langle V_{1}\right.$ : $\left.\tau_{1}, \cdots, V_{k}: \tau_{k}\right\rangle$ (where $\tau_{i}$ is the type of variable $V_{i}$ ). Currently, we only allow types string, int, real, and enum. For an enum type $\tau_{i}$ we assume that a set of values is given, e.g., $\tau_{i}=\{\mathrm{DES}, 3 \mathrm{DES}, \mathrm{AES}\}$.

The set of successors of a node $n$ in a schemata $S$ is denoted by $\operatorname{succ}_{S}(n)$. However, when the schemata $S$ is clear from the context we simply write $\operatorname{succ}(n)$ instead of $\operatorname{succ}_{S}(n)$.

A schemata is shown in Figure 2. The root node is a $\wedge$ node and represented as a square. The left and right child of the root are $\vee$-nodes and represent various authentication and encryption mechanisms respectively. The leaf nodes, such as the ones labeled with none and 3DES, are $V$-nodes with no successors. The special keyword none signifies the fact that an authentication or encryption scheme is not required. Moreover, there are no variables associated with the $\checkmark$-nodes. However, if desired, associated attributes, such as key size for encryption schemes, can be associated with the $\checkmark$-nodes.

Definition 3.2 An instance $I$ of a schemata $S=$ $(N, V$, root $)$ is a subgraph $\left(N^{\prime}, V^{\prime}\right.$, root $)$, where $N^{\prime} \subseteq N$ and $V^{\prime} \subseteq V$. Additionally, following conditions need to be satisfied:

- For a $\wedge$-node $n \in N^{\prime}, \operatorname{succ}(n) \subseteq N^{\prime}$. In other words, all successors of a $\wedge$-node are in the instance $I$.

- For a $\vee$-node $n \in N^{\prime}$, if $\operatorname{succ}(n)$ is non-empty, then $\left|\operatorname{succ}(n) \cap N^{\prime}\right|=1$. In other words, for a $\vee$-node with a non-empty set of successors, exactly one successor is in an instance.
- Consider a node $n \in N^{\prime}$ such that $\operatorname{Var}_{T}(n)=\left\langle V_{1}\right.$ : $\left.\tau_{1}, \cdots, V_{k}: \tau_{k}\right\rangle$. In this case, $I$ assigns values $v_{i}$ of type $\tau_{i}$ to each variable $V_{i}$ in $\operatorname{Var}_{T}(n)$. The tuple of values assigned by $I$ to the node $n$ is denoted by $\operatorname{Val}_{I}(n)$.

Definition 3.3 A policy $P$ for a schemata $S=(N, V$, root $)$ is a 2-tuple $(S, C)$, where $S: N \rightarrow 2^{N}$ and $C$ maps nodes to a tuple of conditions. For each $\vee$-node $n \in N, S(n) \subseteq$ $\operatorname{succ}(n)$, and $C(n)$ is a $k$-tuple of conditions $\left\langle c_{1}, \cdots, c_{k}\right\rangle$ where $\operatorname{Var}(n)=\left\langle V_{1}: \tau_{1}, \cdots, V_{k}: \tau_{k}\right\rangle$. Moreover, we assume that the condition $c_{i}$ applies to values of type $\tau_{i}$. Given a value $v_{i}$ of type $\tau_{i}$, we use $v_{i} \models c_{i}$ to denote that $v_{i}$ satisfies $c_{i}$.

Two policies $P_{1}$ and $P_{2}$ are shown in figures 3 and 4 respectively. Consider the left child of the root. Policy $P_{1}$ specifies that only $\times 509$, Kerberos, and Password are allowed successors for the left node. Other edges and nodes can be interpreted in a similar manner.

Given an instance $I=\left(N^{\prime}, V^{\prime}\right.$, root $)$ and a policy $P=$ $(S, C)$, we say that $I$ satisfies $P$ (denoted by $I \models P$ ) iff the following two conditions are satisfied:

- For all $\vee$-nodes $n \in N^{\prime},\left(\operatorname{succ}(n) \cap N^{\prime}\right) \subseteq S(n)$. In other words, instance $I$ can only choose successors of a $\vee$-node from the subset $S(n)$ provided by the policy $P$.

- Let $\operatorname{Val}_{I}(n)=\left\langle v_{1}, \cdots, v_{k}\right\rangle$ be the values assigned to the node $n$ in $I$, and $C(n)=\left\langle c_{1}, \cdots, c_{k}\right\rangle$ be the conditions assigned to node $n$ by the policy $P$. In this case, for $1 \leq i \leq k, v_{i}=c_{i}$, or each value assigned in the instance $I$ should satisfy the corresponding condition specified by the policy $P$.

Policy $P$ for a schemata $S$ is called satisfiable iff there exists $I$ such that $I \models P$.

Next, we define conjunction of two policies. The conjunction of two policies $P_{1}=\left(S_{1}, C_{1}\right)$ and $P_{2}=\left(S_{2}, C_{2}\right)$ (denoted by $\left.P_{1} \wedge P_{2}\right)$ is a policy $\left(S^{\prime}, C^{\prime}\right)$, where

- For each $\vee$-node $n \in N, S^{\prime}(n)=S_{1}(n) \cap S_{2}(n)$ and $C^{\prime}(n)=\left\langle c_{1}^{1} \wedge c_{1}^{2}, \cdots, c_{k}^{1} \wedge c_{k}^{2}\right\rangle$, where $C_{1}(n)=$ $\left\langle c_{1}^{1}, \cdots, c_{k}^{1}\right\rangle$ and $C_{2}(n)=\left\langle c_{1}^{2}, \cdots, c_{k}^{2}\right\rangle$.

Conjunction of the two example policies $P_{1}$ and $P_{2}$ is depicted in Figure 5

Definition 3.4 A set of $n$ policies $P_{1}, \cdots, P_{n}$ is reconcilable iff there exists an instance $I$ such that $I \models\left(\bigwedge_{i=1}^{n} P_{i}\right)$ or in other words $\bigwedge_{i=1}^{n} P_{i}$ is satisfiable.

Remark: We have described the semantics of reconcilable policies using the satisfaction relation $\models$. One can give an alternative definition in terms of languages. A schemata $S$ 
defines a language of instances $L(S)$, i.e., $L(S)$ contains all instances $I$ of the schemata $I$. A policy $P$ for the schemata $S$ also defines a language of instances $L(P) \subseteq L(S)$, i.e., $L(P)$ contains all instances $I$ such that $I=P$. In this context, policies $P_{i}, \cdots, P_{n}$ are reconcilable iff $\bigcap_{i=1}^{n} L\left(P_{i}\right)$ is non-empty.

\subsection{Resolving multiple partial orders}

Later in this section we discuss policy reconciliation in presence of preferences. In preparation for that, we need to develop some theory about resolving multiple partial orders. Assume that we are given a finite set $S$. Suppose $n$ agents give their preferences on the set $S$, i.e., agent $i$ specifies a partial order $\preceq_{i}$ on the set $S$. Intuitively, an agent $i$ is an organization or process with a policy, and $\preceq_{i}$ specifies the preference of the organization or process. The question is how does one construct a single partial order on the set $S$ (denoted by $\preceq_{1, \cdots, n}$ ) from the $n$ partial orders $\preceq_{1}, \cdots, \preceq_{n}$ ?. Precise definition for combining partial orders is given in [31]. We also provide a a linear time algorithm to compute the combined partial order. For example, consider two partial orders shown in Figure 6 on the set \{kerberos, X509, Password \}. Assuming that the agent giving the partial order $(a)$ has higher preference than the agent with the partial order $(b)$, the combined partial order is $(b)$. Assuming no order between the agents the combined partial order is $(a)$.

\subsection{Reconciliation with preferences}

This section describes reconciliation when policies are allowed to specify preferences. First, we define the concept of policy with preferences.

Definition 3.5 A policy $P$ for a schemata $S=(N, V$, root $)$ is now a 3 -tuple $(S, C$, pref $)$, where $S: N \rightarrow 2^{N}, C$ maps nodes to a tuple of conditions, and pref provides preferences. For each $\vee$-node $n \in N, S(n) \subseteq \operatorname{succ}(n), \operatorname{pref}(n)$ is a partial order on $S(n)$, and $C(n)$ is a $k$-tuple of conditions $\left\langle c_{1}, \cdots, c_{k}\right\rangle$ where $\operatorname{Var}(n)=\left\langle V_{1}: \tau_{1}, \cdots, V_{k}: \tau_{k}\right\rangle$. Moreover, we assume that the condition $c_{i}$ applies to values of type $\tau_{i}$. Given a value $v_{i}$ of type $\tau_{i}$, we assume that $v_{i}=c_{i}$.

A policy $P$ induces a partial order $\preceq_{P}$ on the instances satisfying $P$. Given an instance $I$, the DAG rooted at a node $n$ of $I$ is called a sub-instance, i.e., a sub-instance consists of the node $n$ and all of its descendants. The depth of a sub-instance is the length of the longest path from the root to one of its leaves. The partial order $\preceq_{P}$ is defined on subinstances. Given two sub-instances $S I_{1}=\left(N_{1}, V_{1}\right.$, root $\left._{1}\right)$ and $S I_{2}=\left(N_{2}, V_{2}\right.$, root $\left._{2}\right)$, we say that $S I_{1} \preceq_{P} S I_{2}$ iff the following conditions are satisfied:
- The roots are the same, i.e., $\operatorname{root}_{1}=\operatorname{root}_{2}$.

- root $_{1}$ is a $\wedge$-node.

Let the set of successors of $\operatorname{root}_{1}$ be $\left\{n_{1}, \cdots, n_{k}\right\}$. Let $I_{i}^{1}$ and $I_{i}^{2}$ (for $1 \leq i \leq k$ ) be the sub-instances in $S I_{1}$ and $S I_{2}$ that are rooted at $n_{i}$. In this case the condition is that for all $1 \leq i \leq k, I_{i}^{1} \preceq_{P} I_{i}^{2}$.

- $\operatorname{root}_{1}$ is $a \vee$-node.

Let the successors of root 1 and root $_{2}$ in $S I_{1}$ and $S I_{2}$ be $n_{1}$ and $n_{2}$ respectively, and $I_{n_{1}}$ and $I_{n_{2}}$ be the subinstances rooted at $n_{1}$ and $n_{2}$ respectively. In this case, the condition is the following:

If $n_{1}=n_{2}$, then $I_{n_{1}} \preceq_{P} I_{n_{2}}$; otherwise, $n_{1} \preceq n_{2}$ in the partial order pref $\left(\right.$ root $\left._{1}\right)$ given by the policy $P$.

Notice that $\preceq_{P}$ is inductively defined using the depth of the sub-instances. Intuitively, the partial order $\preceq_{P}$ extends the partial order pref over nodes given by the policy $P$ to subinstances.

Next, we extend the definition of conjunction of two policies to incorporate preferences. The conjunction of two policies $P_{1}=\left(S_{1}, C_{1}\right.$, pref $\left._{1}\right)$ and $P_{2}=\left(S_{2}, C_{2}\right.$, pref $\left._{2}\right)$ (denoted by $\left.P_{1} \wedge P_{2}\right)$ is a policy $\left(S^{\prime}, C^{\prime}\right.$, pref $\left.f^{\prime}\right)$, where

For each $\vee$-node $n \in N, S^{\prime}(n)=S_{1}(n) \cap S_{2}(n)$, pref $^{\prime}(n)$ is equal to $\preceq_{1,2}$, and $C^{\prime}(n)=\left\langle c_{1}^{1} \wedge\right.$ $\left.c_{1}^{2}, \cdots, c_{k}^{1} \wedge c_{k}^{2}\right\rangle$, where $C_{1}(n)=\left\langle c_{1}^{1}, \cdots, c_{k}^{1}\right\rangle$ and $C_{2}(n)=\left\langle c_{1}^{2}, \cdots, c_{k}^{2}\right\rangle$.

Given $n$ reconcilable policies $P_{1}, \cdots, P_{n}$, an instance $I$ is called a most preferred instance or $M P I$ if $I \models\left(\bigwedge_{i=1}^{n} P_{i}\right)$ and $I$ is a maximal element in the partial order induced by the combined policy $\bigwedge_{i=1}^{n} P_{i}$.

\subsection{The Reconciliation Algorithm}

Given $n$ policies $P_{1}, P_{2}, \cdots, P_{n}$, the reconciliation algorithm proceeds as follows:

First, we compute the combined policy $P=\bigwedge_{i=1}^{n} P_{i}$.

Next, starting from the root the combined policy $P$ is traversed recursively to find the most preferred instance according to partial order $\preceq_{P}$ induced by the combined policy.

The complexity of reconciliation algorithm is $O(n)|N|+$ $|E|)$, where $N$ and $E$ are the nodes and edges in $P$. Details of the reconciliation algorithm can be found in [31].

Assume that we are given two policies $P_{1}$ and $P_{2}$ shown in Figures 3 and 4 . The combined policy $P_{1} \wedge P_{2}$ is shown in Figure 5. Suppose that the partial order on authentication mechanisms corresponding to policies $P_{1}$ and $P_{2}$ is 
as shown in Figure 6, and the partial order on the encryption schemes corresponding to the policies $P_{1}$ and $P_{2}$ is as shown in Figure 7. The partial orders are resolved so that policy $P_{1}$ has precedence over policy $P_{2}$. In this case, the partial orders on the authentication and encryption schemes in the combined policy $P_{1} \wedge P_{2}$ is the one corresponding to policy $P_{2}$, i.e., the partial order labeled $(b)$ in the two figures. The MPI computed by our algorithm is shown in Figure 8.

\section{Applications of the policy reconciliation framework}

This section illustrates the use of graphical policy in real application environments. To this end, we show how our policy reconciliation framework can augment IPsec's existing policy negotiation and support the Condor distributed computing system.

\subsection{Graphical Policy in IPsec}

The IPsec [19] suite of protocols provides source authentication, data integrity and data confidentiality at the IP layer. These services are implemented by the Authentication Header (AH) and Encapsulating Security Payload (ESP) transforms. Although not a security service, PCP implements data compression. Each IPsec node (host or security gateway) maintains a security and compression policy defined in terms of these transforms. Communicating peers establish one or more pairs of policy instances (an instance is represented as a security association, or SA) by reconciling configured local policies (called proposals). The Internet Key Exchange protocol (IKE) [16] is used to, among other things, negotiate this governing policy.

IKE policy can be modeled using our graphical approach. To illustrate, suppose that a host desires the following policy:

- All outgoing data must be protected by ESP and $A H$ protocols, and must be compressed using the $P C P$ protocol.

- ESP can use $3 D E S, 3 I D E A$ or DES encryption algorithms, and either HMAC-MD5 or HMAC-SHA integrity/authentication algorithms.

- $A H$ can use either HMAC-MD5 or HMAC-SHA.

- $P C P$ can use either LZS or Deflate.

An IPsec proposal and graphical representation for the example policy is depicted in Figure 9. The hierarchical DAG structure is clearly more expressive and efficient, i.e., one only needs to understand the difference between $\wedge$ (square) and $\vee$ (circle) nodes to interpret policy. Conversely, one needs a great deal of domain knowledge to in- terpret the proposal/transform structure of IPsec. Such intuitive representation simplifies specification, and ultimately reduces policy errors.

Consider an extension to the above policy that states that the use of 3IDEA must use either 128-bit or 256-bit keys. In IPsec, attributes such as key length can be specified only once with each transform. Hence, a separate transform is required for each key length. More generally, the number of transforms grows exponentially in the number of independent attributes. Conversely, the graphical representation only needs to introduce a single subgraph that is shared by the relevant nodes.

\subsection{Hierarchically Policy in the Condor system}

The second example of the policy reconciliation framework is used in the context of Condor [9], a high-throughput distributed system designed to efficiently schedule the usage of distributed and heterogeneous resources such as idle CPU cycles and unused memory. Condor allows resources owners to place various policy requirements on the use of their resources. Our hierarchical DAG structure can succinctly encode Condor security policies. The design of the policy infrastructure and its integration with Condor are detailed in the following section.

\section{Implementation}

We have implemented our hierarchical reconciliation algorithm in the Policy Reconciliation Engine (PRE). PRE reconciles (only) pairs of XML-encoded policies. The restriction of PRE to two-policy reconciliation is not a limitation of our approach, but rather an artifact of the initial target systems' point-to-point communication models (IPSec and Condor). We will extend the implementation to allow multi-party policy reconciliation (e.g., Ismene [23], DCCM [11]) as future needs dictate.

PRE implements an asymmetric requester/responder model. In this model, the requester supplies the relevant policy to the responder. The responder reconciles the received policy with local policies as needed, and the reconciled policy is returned to the requester. Both parties subsequently use the reconciled policy to control the session. We chose a requester/responder model because it most faithfully represents contemporary use of policy (e.g., IKE policy negotiation [16]). This model is similar to client/server communication models. Responders, acting as servers, govern access to the communication resources and requesters, acting as clients, submit requests for those resources. In PRE, the responders assert authority over the resources by placing a higher preference on their own (local) policy. Note that the requester may (and often should) validate that the received reconciled policy is consistent with the origi- 


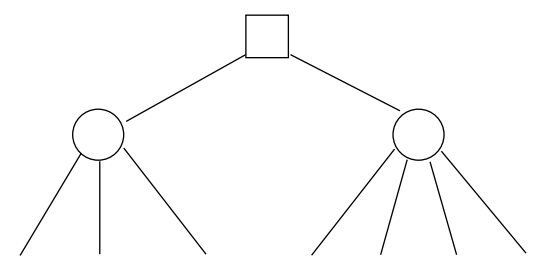

X.509 Kerberos Password none 3DES AES SAFER X.509 Kerberos Password

Figure 3. Example policy

$P_{1}$

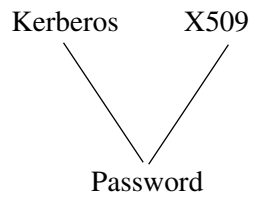

(a)

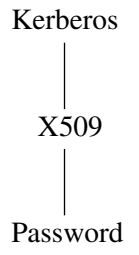

(b)

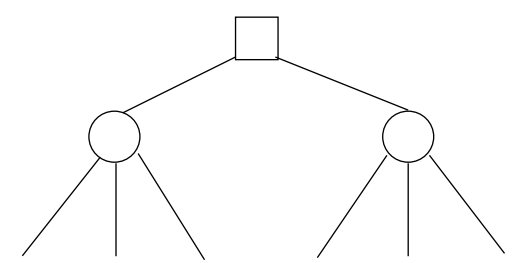

Figure 4. Example policy $P_{2}$

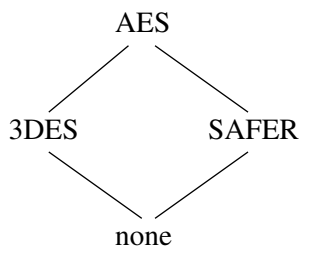

(a)

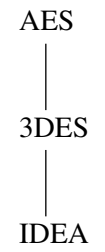

(b)
Figure 6. Two partial orders on authentication schemes.

\section{Figure 7. Two partial orders on encryption schemes.}

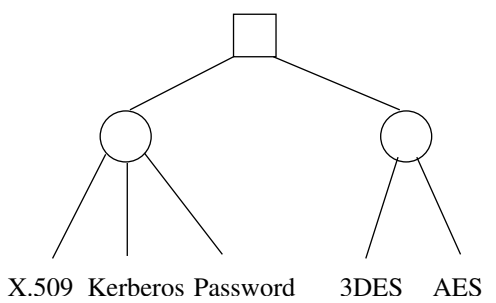

Figure 5. Combined policy $P_{1} \wedge P_{2}$

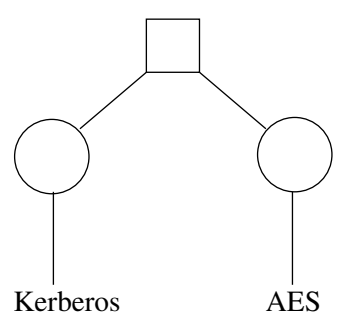

Figure 8. The most preferred instance. nally proposed policy. Policy validation interfaces are provided by PRE.

PRE is both a library and a command line tool. Hence, it can be directly integrated into an application, or used as an external policy processor. Reconciliation is a three-step process in PRE. First, each security policy is parsed into internal data structures. Then each pair of policies is reconciled using the algorithms defined in section 3.3 . Finally, the verification engine ascertains the correctness of the reconciled policy with respect to the local security policy (i.e., implements the consistency test described above).

The current implementation of PRE contains about 1000 lines of $\mathrm{C} / \mathrm{C}++$ code. Source code and documentation for PRE are available for download.

\subsection{Integrating PRE with Condor}

Much of our work in policy has been motivated by the requirements of the Condor system. As described in Section 4.2. Condor schedules resources based on the client requests and other environmental factors. Every Condor peer has a local security policy that governs the services providing the authenticity, confidentiality, and integrity of the session it supports. We have modified the Condor system to use PRE-based reconciliation to construct the security policy used by each session. Past versions of Condor defined security policy using flat structures called Clas$s A d \mathrm{~s}$ [28]. ClassAds flexibly communicate resource advertisements and client requests. However, we found the structure of ClassAds inherently limiting, i.e., we could not represent the appropriate range of acceptable or preferential policies because of their flat structure. Such statements of policy are, as previously argued, hierarchical in nature. This need for hierarchical policy drove our efforts, and ultimately lead to the development of PRE. For details on the implementation, we refer readers to [31].

Currently, Condor does not authenticate the policies or policy exchanges beyond that supported by the underlying transport layer. In general, how and by whom policies are issued and authenticated is an environmental and systems design issue. Environments often require external services for storing and validation of issued policies (e.g., LDAP collections of signed policies). These issues are defined by the larger policy architecture, and is beyond the scope of the current work. Interested readers are referred to [22] for a taxonomy of policy architectures addressing these issues.

\subsection{Performance}

Because of the relatively small policy size and the restriction to pairwise reconciliation, we did not anticipate the introduction of PRE into Condor would significantly impact performance. We sought to measure these costs through several controlled experiments. These experiments measured the total execution time of the policy negotiation protocol defined in the preceding section. All experiments were executed in an environment consisting of a single Central Manager server (333 Mhz duo-processor/Linux RedHat 


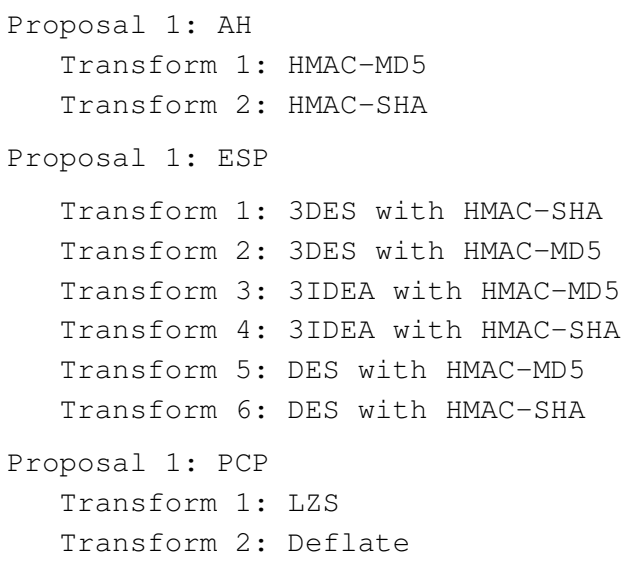

a.) Original IPsec Policy Proposal

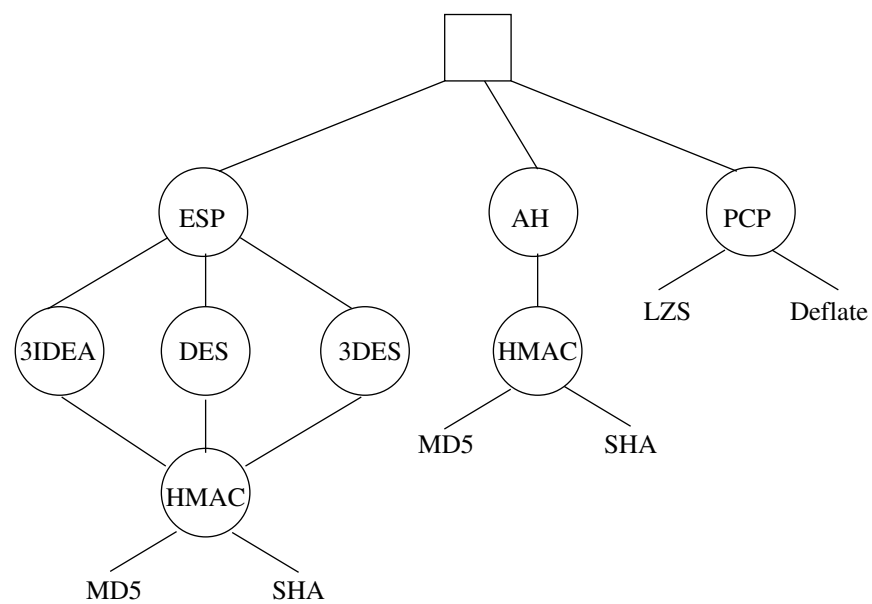

b.) IPsec Policy Schemata in DAG Format

Figure 9. IPsec Policy Example

7.2) and eight clients (three Ultra 10 Sparc Sun/Solaris 2.8 and five $750 \mathrm{MHz}$ Pentium III/Linux Redhat 7.2).

The experimental results confirmed our intuition: the average protocol execution (without $\mathrm{I} / \mathrm{O}$ ), for a policy consisting of authentication, integrity and secrecy, only uses about $5.2 \%$ of the total execution time. When including I/O overhead, the cost is still small-at about $10 \%$ of the overall execution time. Startup cost (i.e. program initialization) is the most dominant factor of the overall execution time, followed closely by overhead incurred from Condor's internal data structures.

\subsection{Future work}

While the theoretical framework and implementation of our hierarchical policy model have reached maturity, we see further exploration of its application to a wide range of problem domains as essential. Initially, we will seek to integrate PRE with widely used policy systems. This will enable us to explore the ways of exploiting the PRE services in specific and policy reconciliation in general. One such work will realize our IPsec policy in software. Integration with tools such as FreeSwan [27] will provide important data-points in the use of extended policy services, and serve to further demonstrate the power of our approach.

We also seek to apply our work to domains which have immediate, but as yet unaddressed, requirements for policy. For example, reconciliation may play an important role in defining security for peer-to-peer (P2P) systems. Currently, there are few coherent security models for P2P. The egalitarian nature of $\mathrm{P} 2 \mathrm{P}$ systems mandate autonomy. Each end-point must be able to assert and realize a set of security requirements deemed important. However, autonomy must be counter-balanced with interoperability. The collec- tion of participants must be able to negotiate a shared view of security. This is precisely the definition of reconciliation. Hence, we claim that the fluid and heterogeneous security models of P2P systems would be well served by our work. Moreover, the clarity and succinctness of hierarchical models may enable more free and open use of security policies in these large communities.

This paper has discussed reconciliation only in the context of security policy. However, hierarchical policy models are applicable to other problem domains. To illustrate, GRID systems share the resources in heterogeneous environments. Participants in the GRID have diverse policies that govern the resource usage. Agreement is often achieved statically in current GRID systems by mandating the adoption of a single universal policy. This mandate is in direct conflict with the needs of dynamic environments whose resource constraints and requirements frequently change. Hence, policy reconciliation systems such as PRE can help to bridge such a gap between dynamicity and the needs for agreement. Furthermore, there is often a direct dependence between resource requirements and security settings and dynamic policy reconciliation can act as the agent between the two. For example, a system that handles sensitive data on remote hosts will require some minimum security policy be enforced.

\section{Conclusion}

Security policy reconciliation is the process of resolving different security policies. In this paper, we presented a formal framework for policy reconciliation. We also presented an efficient algorithm for reconciling different policies. Two distinguishing features of our work are hierarchi- 
cal representation and preferences. We also implemented a simplified version of our algorithm in a software module called PRE and incorporated it in Condor. Experimental results in the context of Condor clearly demonstrate that for each session the reconciliation overhead is negligible.

\section{References}

[1] D. Balenson, D. Branstad, P. Dinsmore, M. Heyman, and C. Scace. Cryptographic Context Negotiation Protocol. Technical report, Network Associates, Inc., 1999.

[2] D. Balenson, D. Branstad, D. McGrew, J. Turner, and M. Heyman. Cryptographic Context Negotiation Template. Technical report, Network Associates, Inc., 1999.

[3] Y. Bartal, A. J. Mayer, K. Nissim, and A. Wool. Firmato: A novel firewall management toolkit. In IEEE Symposium on Security and Privacy, pages 17-31, 1999.

[4] D. Bell and L. LaPadula. Secure Computer Systems: Mathematical Foundations and Model. Technical Report M74-244, MITRE Corperation, Bedford, MA, 1973.

[5] M. Blaze, J. Feigenbaum, and A. Keromytis. KeyNote: Trust management for public-key infrastructures. Lec. Notes in Comp. Sci., 1550:59-63, 1999.

[6] M. Blaze, J. Feigenbaum, and J. Lacy. Decentralized Trust Management. In Proceedings of the 1996 IEEE Symposium on Security and Privacy, pages 164-173, November 1996. Los Alamitos.

[7] M. Blaze, J. Ioannidis, and A. D. Keromytis. Trust management for IPsec. Information and System Security, 5(2):95118, 2002.

[8] L. Cholvy and F. Cuppens. Analyzing Consistency of Security Policies. In 1997 IEEE Symposium on Security and Privacy, pages 103-112. IEEE, May 1997. Oakland, CA.

[9] Condor. http://www.cs.wisc.edu/condor/.

[10] J. DeTreville. Binder, a logic-based security language. In Symp. on Res. in Sec. and Privacy, Oakland, CA, May 2002. IEEE Computer Society Press.

[11] P. Dinsmore, D. Balenson, M. Heyman, P. Kruus, C. Scace, and A. Sherman. Policy-Based Security Management for Large Dynamic Groups: An Overview of the DCCM Project. In DARPA Information Survivability Conference and Exposition, pages 64-73, 2000.

[12] C. Ellison. SPKI requirements. RFC 2692, Sept. 1999.

[13] C. Ellison, B. Frantz, B. Lampson, R. L. Rivest, B. Thomas, and T. Ylonen. SPKI certificate theory. RFC 2693, Sept. 1999.

[14] Z. Fu and S. F. Wu. Automatic Generation of IPSec/VPN Security Policies In an Intra-Domain Environment. In Proceedings of the 12th International Workshop on Distributed System Operation \& Management (DSOM 2001), pages 279290, October 2001.

[15] L. Gong and X. Qian. The Complexity and Composability of Secure Interoperation. In Proceedings of the IEEE Symposium on Research in Security and Privacy, pages 190-200, Oakland, California, May 1994. IEEE.

[16] D. Harkins and D. Carrel. The Internet Key Exchange (IKE). http://www.ietf.org/rfc/rfc2409.txt, 1998.

[17] J. Hoagland, R. Pandey, and K. Levitt. Security Policy Specification Using a Graphical Approach. Technical Report CSE-98-3, The University of California, Davis Department of Computer Science, June 1998.
[18] T. Jim. SD3: A trust management system with certified evaluation. In Proceedings of the 2001 IEEE Symposium on Security and Privacy, May 2001.

[19] S. Kent and R. Atkinson. Security Architecture for the Internet Protocol. http://www.ietf.org/rfc/rfc2401.txt, 1998.

[20] M. Koch, L. V. Mancini, and F. Parisi-Presicce. A GraphBased Formalism for RBAC. Transactions on Information and System Security (TISSEC), 5(3):332 - 365, 2002.

[21] M. Litzkow, M. Livny, and M. Mutka. Condor - A Hunter of Idle Workstations. In Proceedings of the 8th International Conference of Distributed Computing Systems (ICDCS), pages 104-111, 1988.

[22] P. McDaniel. Policy Management in Secure Group Communication. PhD thesis, University of Michigan, Ann Arbor, MI, August 2001.

[23] P. McDaniel and A. Prakash. Methods and limitations of security policy reconciliation. In 2002 IEEE Symposium on Security and Privacy, pages 73-87, May 2002.

[24] P. McDaniel, A. Prakash, and P. Honeyman. Antigone: A Flexible Framework for Secure Group Communication. In Proceedings of the 8th USENIX Security Symposium, pages 99-114, August 1999.

[25] M. Nyanchama and S. Osborn. The Role Graph Model and Conflict of Interest. Transactions on Information and System Security (TISSEC), 2(1):3 - 33, 1999.

[26] L. Pearlman, V. Welch, I. Foster, C. Kesselman, and S. Tuecke. A Community Authorization Service for Group Collaboration. In Proceedings of the IEEE 3rd International Workshop on Policies for Distributed Systems and Networks, 2001.

[27] T. F. Project. Linux FreeS/WAN. http://www.freeswan.org/.

[28] R. Raman, M. Livny, and M. Solomon. Matchmaking: Distributed Resource Management for High Throughput Computing. In Proceedings of the Seventh IEEE International Symposium on High Performance Distributed Computing (HPDC), 1998.

[29] R. S. Sandhu, E. J. Coyne, H. L. Feinstein, and C. E. Youman. Role-Based Access Control Models. IEEE Computer, 29(2):38-47, 1996.

[30] R. S. Sandhu and P. Samarati. Access Control: Principles and Practice. IEEE Communications Magazine, 32(9):4048, 1994.

[31] H. Wang, S. Jha, P. McDaniel, and M. Livny. Security Policy Reconciliation in Deistributed Computing Environments. Technical Report 1499, University of Wisconsin-Madison, March 2004.

[32] Y. Yang, Z. Fu, and S. F. Wu. BANDS: An Inter-domain Internet Security Policy Management System for IPSec/VPN. In Integrated Network Management, volume 246 of IFIP Conference Proceedings, pages 231-244. Kluwer, 2003.

[33] J. Zao, L. Sanchez, M. Condell, C. Lynn, M. Fredette, P. Helinek, P. Krishnan, A. Jackson, D. Mankins, M. Shepard, and S. Kent. Domain Based Internet Security Policy Management. In DARPA Information Survivability Conference and Exposition, pages 41-53, 2000. 